\title{
Clinical Utility of a Magnetic Resonance-conditional Pacemaker in a Patient with Cardiac Sarcoidosis
}

\author{
Kazuya Ishibashi, Mitsuo Takeda and Yasuhiro Yamahara
}

\begin{abstract}
We herein present a case of cardiac sarcoidosis with atrioventricular (AV) block that was evaluated using magnetic resonance imaging (MRI) before and after pacemaker implantation. An echocardiogram showed wall thinning in the basal septum. MRI showed late gadolinium enhancement in the interventricular septum and right ventricle. Fluorine-18-fluorodeoxyglucose positron emission tomography (PET) demonstrated abnormal uptake in the same area. An MR-conditional pacemaker was implanted to treat AV block. Steroid treatment resulted in the remission of the cardiac lesions and AV block, as confirmed by PET and MRI. MRconditional pacemakers are thus considered to have great advantages in treating cardiac sarcoidosis with AV block.
\end{abstract}

Key words: sarcoidosis, pacemaker, magnetic resonance imaging, atrioventricular block, ST-segment elevation, positron emission tomography

(Intern Med 52: 1341-1345, 2013)

(DOI: 10.2169/internalmedicine.52.9364)

\section{Introduction}

Sarcoidosis is a systemic disease of unknown etiology characterized by the formation of noncaseating epithelioid cell granulomas (1). The severity of cardiac involvement is regarded to be a key factor in the prognosis of patients with sarcoidosis. In patients with cardiac sarcoidosis, magnetic resonance imaging (MRI) is essential for evaluating the expansion of myocardial lesions $(2,3)$. In Japan, however, it is impossible to perform magnetic resonance (MR) scans in any patient with a cardiac electronic device due to the absolute contraindication of MRI for such patients. Recently, MR-compatible pacemakers have become available in Japan. We herein report a case of cardiac sarcoidosis with complete atrioventricular (AV) block that was evaluated using MRI both before and after pacemaker implantation.

\section{Case Report}

A 55-year-old woman was referred to our outpatient cardiology clinic in 2011 for an evaluation of electrocardiographic abnormalities. She was asymptomatic at the first visit and had no history of syncope. No sudden deaths were reported in her family. A 12-lead electrocardiogram (ECG) showed complete right bundle branch block (CRBBB) and right axis deviation accompanied by ST-segment elevation in leads V1 through V3, mimicking Brugada syndrome (Fig. 1-A). An echocardiogram demonstrated a normal cardiac function with mild thickening of the interventricular septum (13 mm) (Fig. 1-A). No abnormalities were detected on a treadmill exercise test or holter ECG. Therefore, we recommended an annual follow-up. After one year (in 2012), the patient experienced easy fatigability and exertional dyspnea. An ECG showed bradycardia due to complete AV block with junctional escape beats (Fig. 1-B). An echocardiogram indicated focal thinning $(7 \mathrm{~mm})$ and hypokinesis in the basal interventricular septum, although the global left ventricular (LV) function was maintained, with a left ventricular ejection fraction of $65 \%$ (Fig. 1-B). MRI scans (Vantage Titan, Toshiba, 1.5-Tesla system) also revealed severe hypokinesis and wall thinning in the basal interventricular septum. Moreover, patchy late gadolinium (Gd) enhancement was clearly observed in the interventricular septum and right ventricular free wall (Fig. 2-A). A gallium-67 $\left({ }^{67} \mathrm{Ga}\right)$ citrate scintigram revealed slight uptake in 
(A)
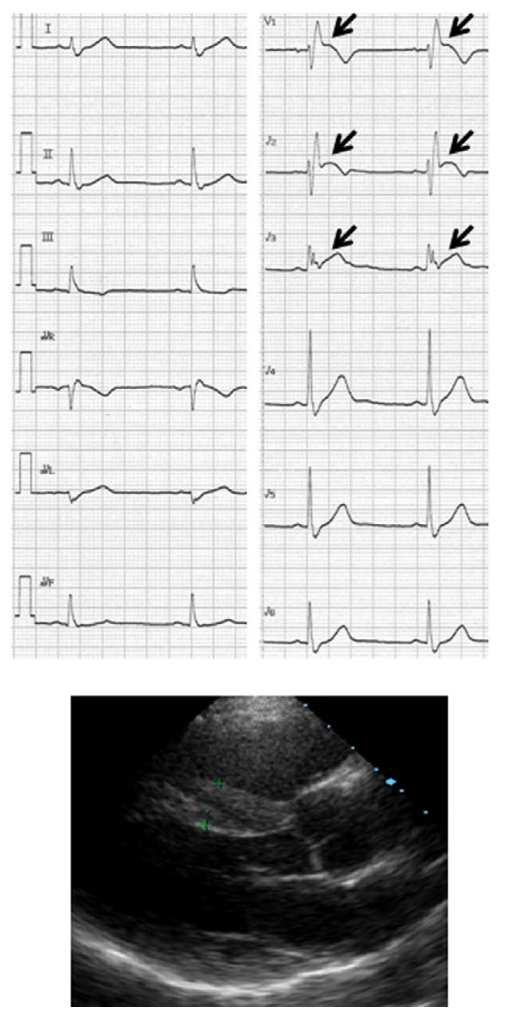

(B)
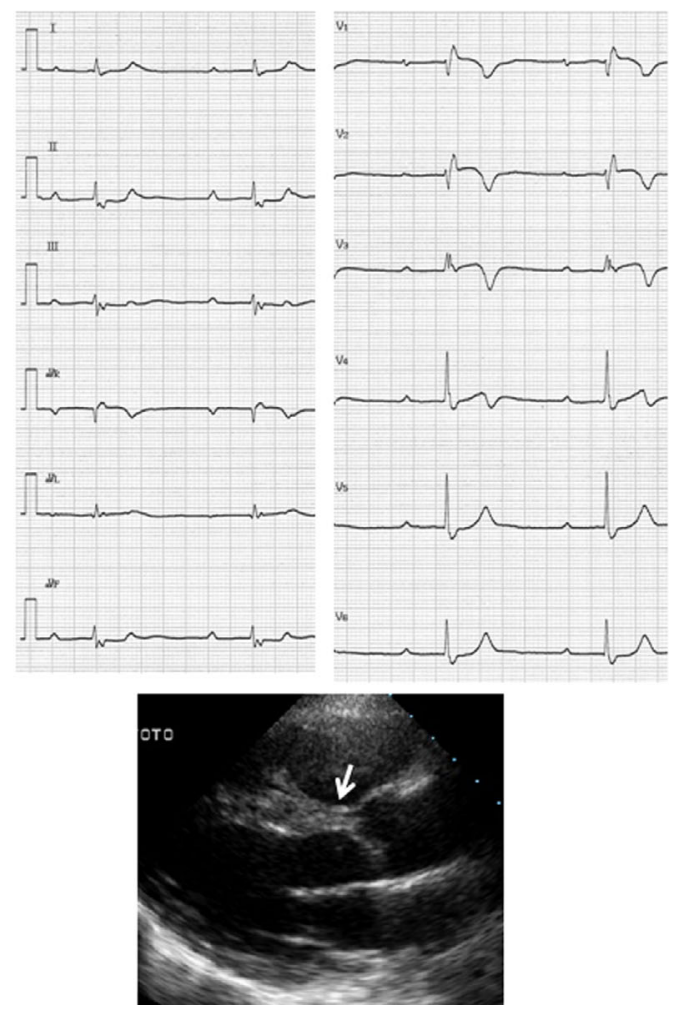

Figure 1. Twelve-lead electrocardiograms and echocardiograms obtained at the first and second (one year later) clinic visits. Note that $S T$-segment elevation is visible in leads $V_{1}$ through $V_{3}$ (black solid arrows). The white solid arrow indicates wall thinning in the basal portion of the interventricular septum.

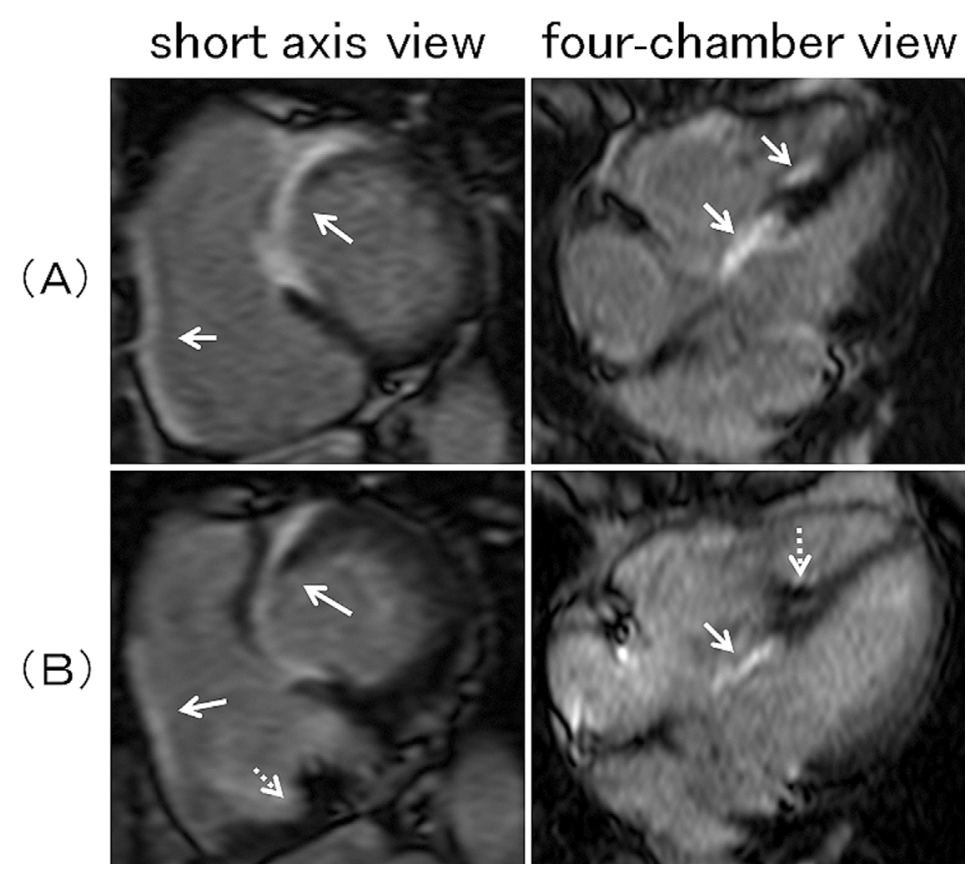

Figure 2. Magnetic resonance imaging studies conducted before and after steroid therapy. The white solid arrows show late gadolinium enhancement in the interventricular septum and right ventricular free wall. Following steroid treatment with pacemaker therapy, the late gadolinium-enhanced area diminished in size. Note that the pacemaker lead is observed in the right ventricle and that the tip of the lead is positioned in the middle septum (white dotted arrows). 

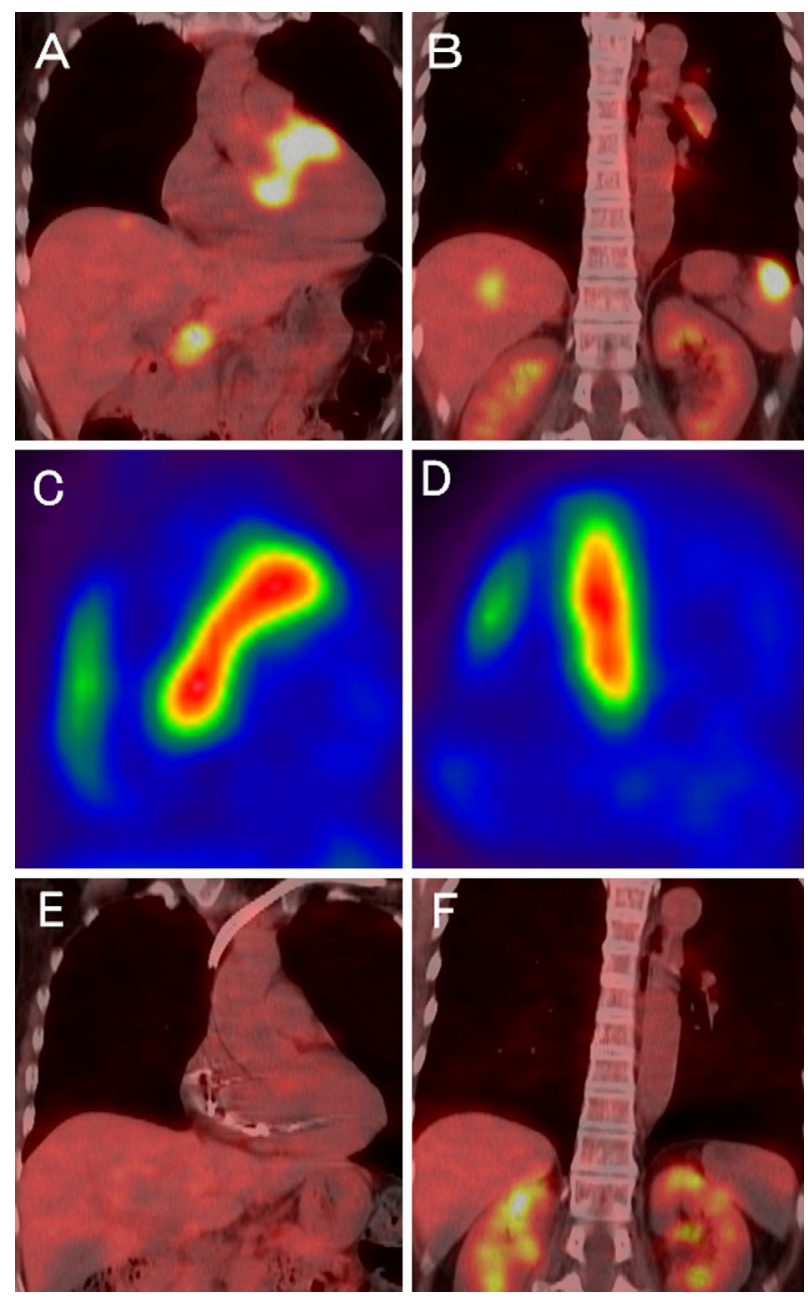

Figure 3. Fluorine-18-fluorodeoxyglucose $\left({ }^{18}\right.$ F-FDG) positron emission tomography/computed tomography scans obtained before and after steroid therapy. (A) and (B): Abnormal uptake of ${ }^{18}$ F-FDG was registered in the heart, intraabdominal lymph nodes, liver and spleen before treatment. (C) Short axis view and (D) 4-chamber view: In the heart, abnormal uptake was located in the interventricular septum and right ventricular free wall. (E) and (F): Abnormal uptake of ${ }^{18}$ F-FDG was entirely eliminated by steroid therapy.

the heart. Fluorine-18-fluorodeoxyglucose $\left({ }^{18} \mathrm{~F}-\mathrm{FDG}\right)$ positron emission tomography (PET)/computed tomography (CT) indicated obvious hot spots in the heart, spleen, liver and intra-abdominal lymph nodes (Fig. 3-A, B). In the heart, an abnormal uptake of ${ }^{18}$ F-FDG was detected in the interventricular septum and right ventricular free wall (Fig. 3-C, D). A technetium-99m ( $\left.{ }^{99 \mathrm{~m}} \mathrm{Tc}\right)$-tetrofosmin scintigram showed decreased uptake in the basal interventricular septum. An ocular examination was normal. A tuberculin reaction test was negative. The serum levels of angiotensinconverting enzyme and lysozyme were within the normal ranges. Angiography indicated normal coronary arteries. An endomyocardial biopsy was obtained from the right ventricular septum, the results of which indicated nonspecific abnormalities, including mild interstitial edema and mild lymphocytic infiltration. According to the diagnostic stan- dards of the Japan Society of Sarcoidosis and Other Granulomatous Disorders (4), the patient was diagnosed as having sarcoidosis with cardiac involvement despite the lack of histopathological evidence. In view of the patient's ongoing need for MRI follow-up, an MR-conditional pacemaker (Advisa MRI $^{\circledR}$ A3DR01, Medtronic) and leads (CapSureFix MRI $^{\circledR}$ 5086MRI-45, 5086MRI-52, Medtronic) were implanted (Fig. 4). After the absence of any inflammatory signs around the pacemaker pocket was confirmed, oral prednisolone was initiated at a dose of $30 \mathrm{mg} /$ day. Four weeks later, the patient's AV block persisted, although her symptoms were relieved. Six weeks later, an ECG revealed recovery of AV conduction. Therefore, the dose of prednisolone was tapered. Three months later, PET/CT and cardiac MRI were conducted to evaluate the efficacy of the steroid treatment. ${ }^{18} \mathrm{~F}$-FDG PET/CT revealed no abnormal uptake, which suggested the effectiveness of the steroid therapy (Fig. 3-E, F). Before the MRI procedure, the device was evaluated. No problems were observed in the pacemaker system. The device was subsequently switched to SureScan ${ }^{\circledR}$ mode, which is compatible with MRI. Subsequently, ODO mode (i.e., nonpacing mode) was chosen. MRI was performed in the normal operating mode because the use of a first level controlled operating mode is not permitted in patients with pacemakers. During the MRI scan, the patient was monitored continuously with pulse oximetry and ECG. After MR scanning, the pacemaker system was confirmed to be normal then reprogrammed to the previous mode. The MRI scan revealed a decreased area of late Gd enhancement (Fig. 2-B).

\section{Discussion}

The primary finding of this case report is that MRconditional pacemakers are of great value in the management of patients with AV block caused by cardiac sarcoidosis.

This patient was diagnosed to have cardiac sarcoidosis based on the following findings: (1) wall thinning in the basal interventricular septum; (2) progressive heart block (complete atrioventricular block); (3) an abnormal uptake of ${ }^{67} \mathrm{Ga}$ citrate and ${ }^{18} \mathrm{~F}-\mathrm{FDG}$ in the myocardium; (4) a decreased uptake of ${ }^{99 \mathrm{~m}} \mathrm{Tc}$-tetrofosmin in the basal interventricular septum; (5) patchy late Gd enhancement in the myocardium on MRI scans; and (6) negative tuberculin reaction test results. Three items (1), (2) and (3) fulfilled the major diagnostic criteria for cardiac sarcoidosis (4). We later confirmed that the patient's ECG obtained in 2009 was normal, two years before her first visit to our clinic. In 2010, one year before her first clinic visit, her ECG showed CRBBB without STsegment elevation. Interestingly, the ECG showed CRBBB with ST-segment elevation resembling Brugada syndrome at presumably an early stage of sarcoidosis (in 2011), when no significant structural abnormalities other than mild septal thickening were detected on an echocardiogram. The STsegment elevation observed in the right precordial leads may 


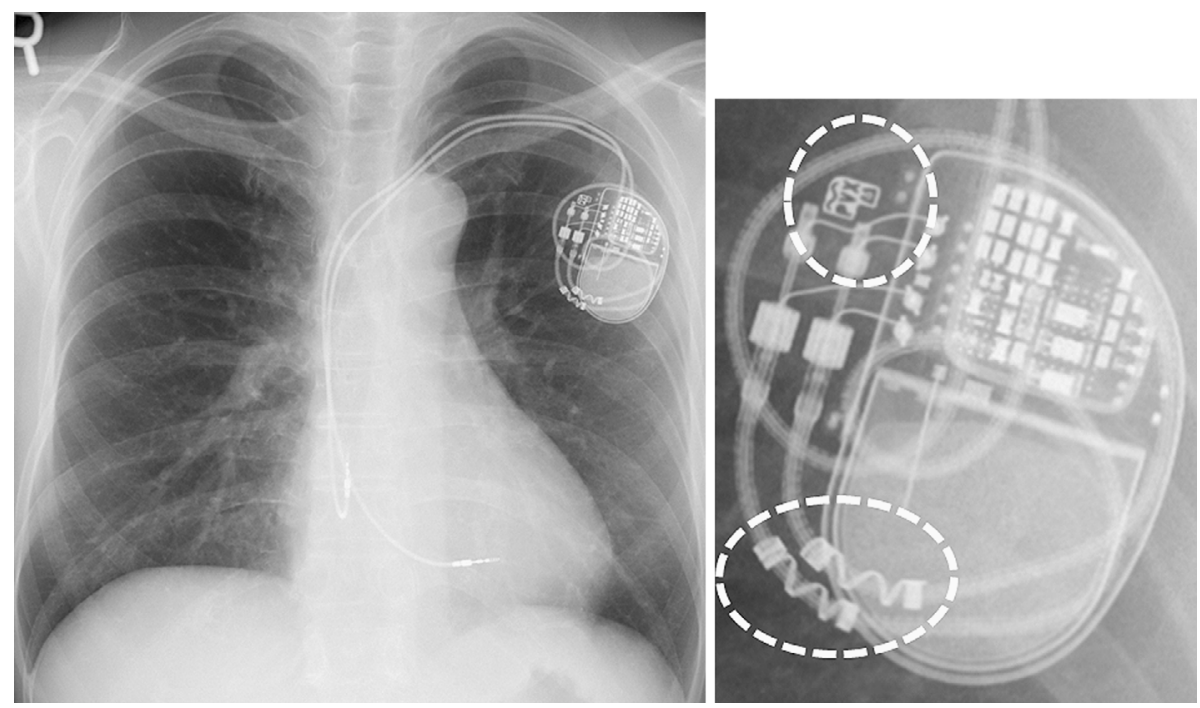

Figure 4. Chest radiography showing an implanted magnetic resonance (MR)-conditional pacemaker. A dual-chamber pacemaker implanted in the left chest wall. The ventricular lead positioned in the low right septum. Notable features of the MR-conditional device system (the generator and leads) are circled.
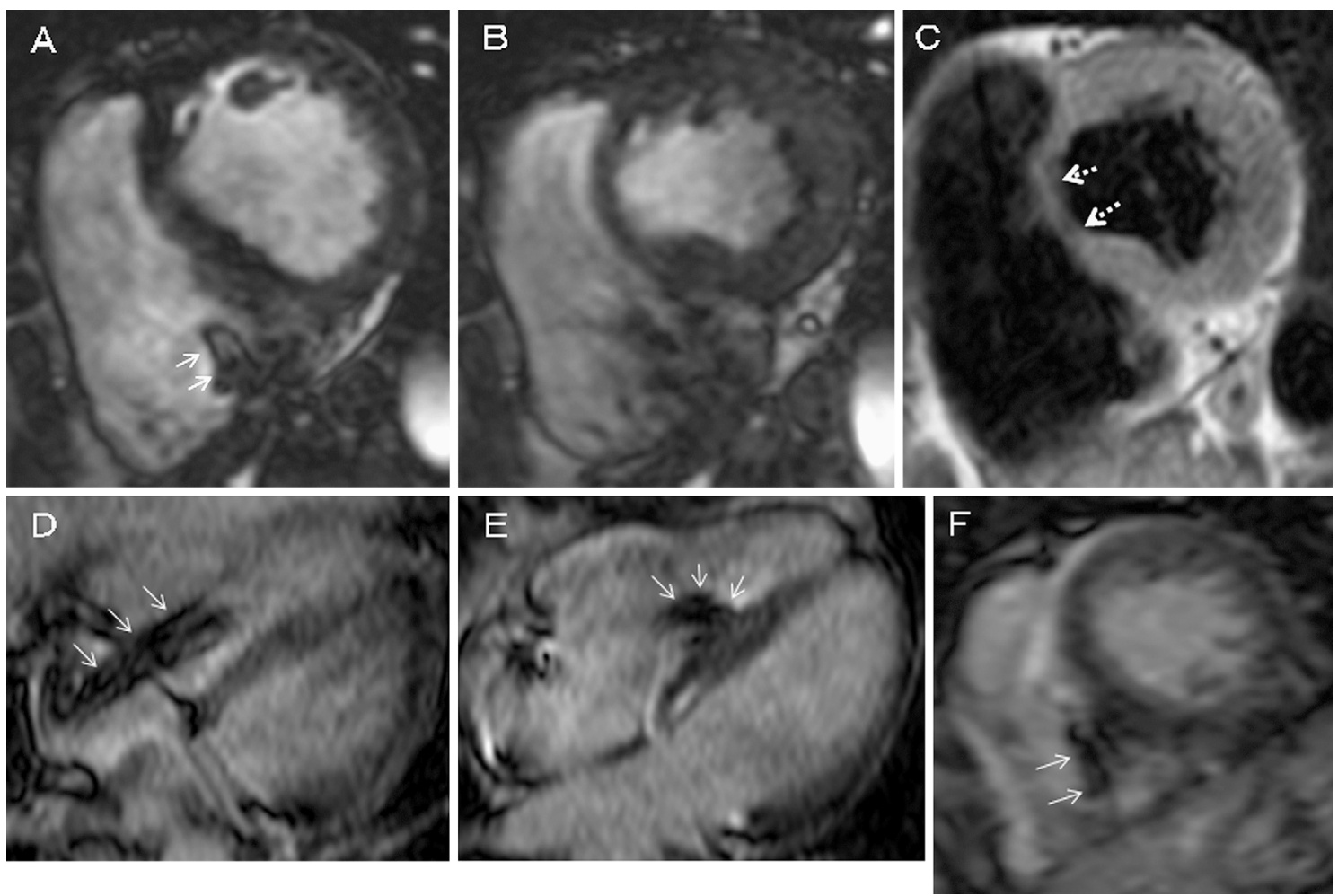

Figure 5. Cardiac magnetic resonance scan images of the pacemaker system. (A) and (B): Cine imaging (end-diastolic and end-systolic phase). (C): T2-weighted imaging with dark blood preparation and fat suppression. (D), (E) and (F): Inversion recovery fast field echo. Wall thinning and dyskinesis are observed in the basal interventricular septum (dotted arrows). Note that the pacemaker leads are visible in the right atrium and ventricle (solid arrows). The tip of the RV pacing lead is located in the middle septum.

thus have been caused by transmural inflammation in the interventricular septum.

In the present case, ${ }^{18} \mathrm{~F}-\mathrm{FDG}$ PET/CT was more valuable in assessing the disease activity than ${ }^{67} \mathrm{Ga}$ citrate scintigra- phy, as has been reported by many investigators $(5,6)$. In our case, a PET/CT scan revealed active lesions in the liver, spleen and lymph nodes that were not detected on a ${ }^{67} \mathrm{Ga}$ citrate scintigram. Moreover, PET/CT demonstrated the ex- 
tent of myocardial lesions and the effectiveness of the steroid treatment.

Cardiac MRI is a useful modality for detecting myocardial inflammation, edema, fibrosis, and fat infiltration (7). Therefore, MRI plays an important role in the diagnosis and management of cardiac sarcoidosis (2). In particular, the presence of late $\mathrm{Gd}$ enhancement is important for detecting sarcoid lesions. In our case, the segments of late $\mathrm{Gd}$ enhancement were similar to those of abnormal cardiac uptake of ${ }^{18} \mathrm{~F}$-FDG. Therefore, the late Gd-enhanced segments were regarded as active lesions. Until recently, it was an absolute contraindication for patients with pacemakers to undergo MRI, despite the usefulness of MRI in diagnosing sarcoidosis patients with AV block. Since October 2012, however, MR-conditional pacemakers have become available in Japan. Importantly, the MR-conditional pacemaker system consist of an MR-conditional generator, MR-conditional leads and MR-conditional programming (8). That is, we should note that the safety of MRI is guaranteed only under the condition that the complete system is used. Moreover, during MRI scanning in patients with this pacemaker, only the 1.5Tesla system with the normal operating mode is available. Consequently, in patients with pacemakers, the quality of MR images is likely to be lower than usual. In our case, however, the restricted MR operating mode had little influence on the quality of the MR scan images.

To the best of our knowledge, there has been only one case report (in the UK) of cardiac MRI performed on a sarcoidosis patient with an MR-conditional pacemaker (9). In our case, the presence of late Gd enhancement showed that the myocardial lesions had diminished in size following steroid treatment. Considering both the PET and MRI scan findings, the remaining area of $\mathrm{Gd}$ enhancement was likely to be inactive and scarred. The quality of other MR scan im- ages was not impaired, even with the pacemaker (Fig. 5). MRI demonstrated that the tip of the right ventricular lead was positioned in the middle septum near the area of late Gd enhancement. Therefore, we must be cautious of future rising of the pacing threshold. MRI therefore appears to have some advantages over other modalities in the assessment of the lead position and myocardial lesions.

The authors state that they have no Conflict of Interest (COI).

\section{References}

1. Iannuzzi CM, Rybicki AB, Teirstein SA. Sarcoidosis. N Engl Med 357: 2153-2165, 2007.

2. Smedema JP, Snoep G, van Kroonenburgh MP, et al. Evaluation of the accuracy of gadolinium-enhanced cardiovascular magnetic resonance in the diagnosis of cardiac sarcoidosis. J Am Coll Cardiol 45: 1683-1690, 2005.

3. Patel MR, Cawley PJ, Heitner JF, et al. Detection of myocardial damage in patients with sarcoidosis. Circulation 120: 1969-1977, 2009.

4. Diagnostic standard and guidelines for sarcoidosis. Jpn J Sarcoidosis and Granulomatous Disorders 27: 89-102, 2007.

5. Okumura W, Iwasaki $T$, Toyama $T$, et al. Usefulness of fasting 18F-FDG PET in identification of Cardiac Sarcoidosis. J Nucl Med 45: 1989-1998, 2004.

6. Nishiyama Y, Yamamoto Y, Fukunaga K, et al. Comparative evaluation of ${ }^{18} \mathrm{~F}$-FDG PET and ${ }^{67} \mathrm{Ga}$ scintigraphy in patients with sarcoidosis. J Nucl Med 47: 1571-1576, 2006.

7. Wassmuth R. Cardiac magnetic resonance imagings for myocarditis and nonischemic cardiomyopathies. Minerva Cardioangiol 57: 511-520, 2009.

8. Wilkoff LB, Bello D, Taborsky D, et al. Magnetic resonance imaging in patients with a pacemaker system designed for the magnetic resonance environment. Heart Rhythm 8: 65-73, 2011.

9. Quarta G, Holdright RD, Plant TG, et al. Cardiovascular magnetic resonance in cardiac sarcoidosis with MR conditional pacemaker in situ. J Cardiovasc Magn Reson 13: 26, 2011.

(C) 2013 The Japanese Society of Internal Medicine http://www.naika.or.jp/imonline/index.html 